\title{
ANÁLISIS DEL SISTEMA DE GESTIÓN AMBIENTAL BASADO EN LA NORMA TÉCNICA COLOMBIANA ISO 14001:2015 PARA LAS EMPRESAS QUE REALICEN LA FRACTURACIÓN HIDRÁULICA PARA LA EXPLOTACIÓN DE GAS DE LUTITAS
}

\author{
ANALYSIS OF ENVIRONMENTAL MANAGEMENT SYSTEM REQUIREMENTS BASED \\ COLOMBIAN TECHNICAL STANDARD ISO 14001:2015 PLACING FOR COMPANIES \\ FOR FRACKING SHALE GAS EXPLOITATION
}

Yenith Cristina Ortiz González*

Cristian Rene Rodríguez Sarmiento**

Leidy Carolina Ramírez Moya***

Recibido: 11 de enero de 2017

Aceptado: 25 de abril de 2017

\section{Resumen}

El artículo analiza la NTC ISO 14001: 2015 para las empresas que realizan fracturación hidráulica en yacimientos de lutitas en Colombia. Se utilizaron los requisitos de la norma NTC ISO 14001: 2015 aplicados a este tipo de empresas como fundamento metodológico. Se encontró que si bien la fracturación hidráulica puede traer beneficios para el sector petrolero, técnica que aumenta los impactos ambientales; de manera que la implementación de un sistema de gestión ambiental no es suficiente para controlar los impactos ambientales generados por la práctica, factor que cuestiona el papel de las organizaciones frente a la gestión de sus actividades.

Palabras clave: fracturación hidráulica, yacimientos no convencionales, gas de lutita, NTC ISO 14001: 2004, sistemas de gestión ambiental.

\section{Abstract}

This paper aims to analyze the NTC ISO 14001: 2015 for companies engaged in hydraulic fracturing in shale deposits in Colombia. As a methodology, the requirements of the ISO 14001: 2015 standard applied to this type of company were used. The hydraulic fracturing technique benefits within the petroleum sector were found. However, the implementation of an environmental man-

\footnotetext{
* Ingeniera industrial, especialista en Gerencia de Productividad, magíster en Calidad y Gestión Integral. Estudiante de Doctorado en Educación. Docente investigadora, Centro de Investigación en Competitividad Empresarial, Fundación Universidad de América. induscris@yahoo.es yenith.ortiz@investigadores.uamerica.edu.co

** Ingeniero de petróleos, especialista en Gerencia de la Calidad. Centro de Investigación en Competitividad Empresarial, Fundación Universidad de América. crrodriguezs27@gmail.com

${ }^{* * *}$ Ingeniera química, especialista en Gerencia de la Calidad. Centro de Investigación en Competitividad Empresarial, Fundación Universidad de América. leidy.ramirez@estudiantes.uamerica.edu.co
} 
agement system is not enough to control the environmental impacts generated by the practice, questioning the role of organizations in the management of their activities.

Keywords: Hydraulic fracturing, unconventional reservoirs, Shale Gas, NTC ISO 14001: 2004 Environmental Management Systems.

\section{INTRODUCCIÓN}

Actualmente, un alto porcentaje del petróleo y el gas producidos en el mundo proviene de yacimientos convencionales. Debido a que los hidrocarburos son recursos no renovables se debe buscar nuevos yacimientos para cumplir con la demanda actual, almacenar y abastecer la economía del sector en un corto plazo. Los yacimientos más complejos (denominados yacimientos no convencionales) se convierten en una solución para aumentar las reservas y explotar nuevos recursos, y son un reto de ingeniería e innovación tecnológica. En Colombia, la actividad petrolera es uno de los mayores contribuyentes a la economía nacional. La figura 1 muestra el aporte del sector al producto interno bruto (PIB).

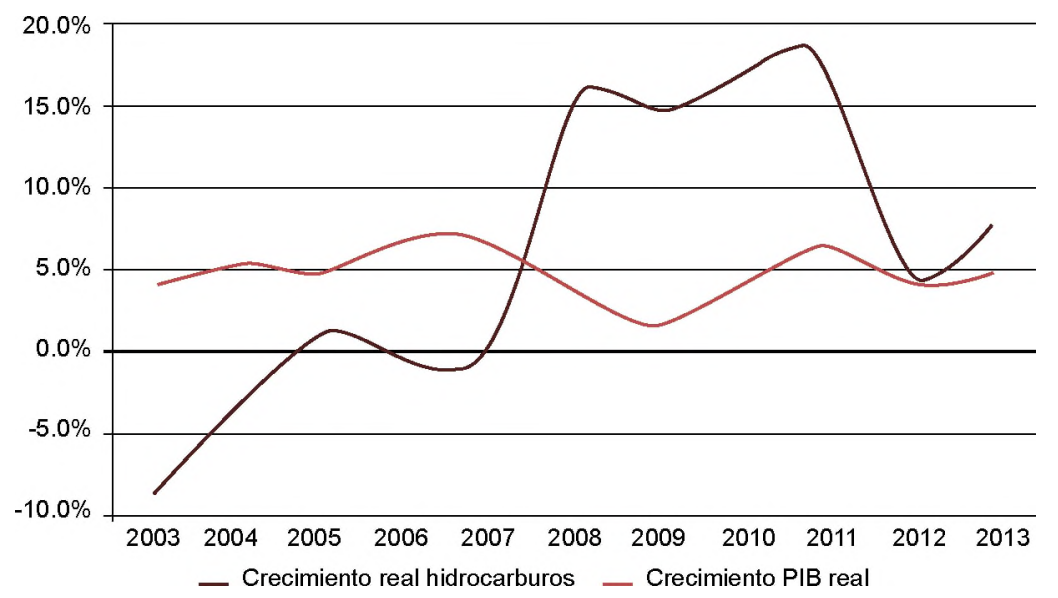

a) Crecimiento valor agregado de hidrocarburos vs. crecimiento PIB Real

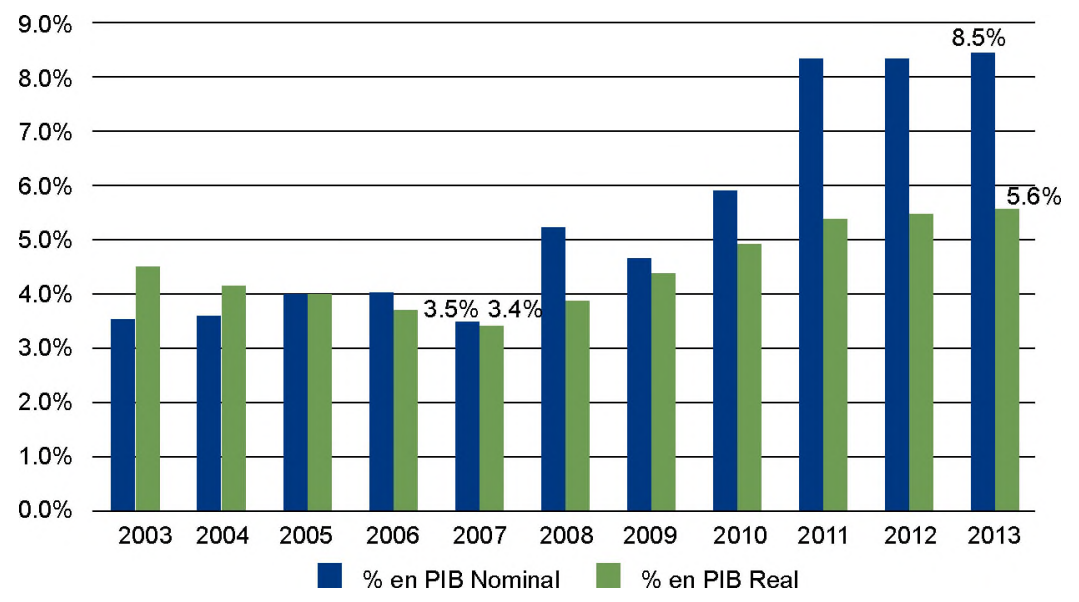

b) Porcentaje del valor agregado de los hidrocarburos en el PIB

Figura 1. Crecimiento y participación del sector petrolero en la economía.

Fuente: Unidad de planeación Minero Energética (2015). 
En la figura 1 se observa que desde 2008 el sector de hidrocarburos ha crecido más rápido que el PIB total. Mientras que la economía colombiana en los últimos diez años lo hizo a un ritmo de $4.8 \%$, el sector de hidrocarburos logró el $7.1 \%$ anual, lo que ha llevado a un aumento paulatino de la participación de la actividad petrolera en el producto total de la economía colombiana. En el año 2013, el sector contribuyó con 5.6 \% del total del PIB real, lo que representa un incremento de $65 \%$ desde 2007 , periodo que registró una participación de $3.4 \%$. De acuerdo con la Unidad de Planeación Minero Energética (2015), el crecimiento en el sector petrolero, en términos de pesos corrientes, se intensificó desde el año 2011, pues superó la barrera del $8 \%$ y generó para 2013 un valor agregado de $\$ 59.7$ billones de pesos (US\$32 000 millones).

Por otro lado, la industria petrolera se encuentra en una revolución tecnológica para mantener y aumentar la producción de hidrocarburos y dar cumplimiento a la demanda energética. Ferrari (citado en Robles, 2004, p. 24) asegura que la industria petrolera tiene la tarea de tomar decisiones importantes sobre los métodos de producción, lo que obliga a los profesionales a estudiar unidades geológicas con características distintas a los yacimientos convencionales (que posteriormente serán evaluados y explotados).

Uno de los métodos más utilizados para los yacimientos no convencionales es la fracturación hidráulica de lutitas. El procedimiento consiste en la inyección de un fluido a presiones considerablemente altas sobre un yacimiento con alto potencial de contener hidrocarburos (se utiliza generalmente agua mezclada con productos químicos); después de la disminución de presión, se usa arena o un material soporte que se aloja dentro de las grietas de las rocas para evitar su cierre, permitiendo que el gas y el petróleo circulen hacia el pozo y salgan la superficie (Bertinat et ál., 2014).

Para el caso colombiano, los yacimientos no convencionales de shale gas beneficiarían en gran medida al sector. De acuerdo con Colombia Energía (2012), la industria petrolera aportaría al suministro energético la autosuficiencia de gas natural y las posibilidades de exportación. Lo anterior demuestra que la exploración de este tipo de yacimiento representaría un avance en términos económicos, tecnológicos e industriales, que además tendría una afectación indirecta y positiva a la economía nacional.

Sin embargo, existe un debate mundial respecto a la viabilidad ambiental de esta técnica; tanto así que hay países que la han prohibido sin importar el potencial de hidrocarburos no convencionales recuperables que se han estimado. La tabla 1 ilustra los riesgos asociados a la fracturación hidráulica.

Tabla 1. Riesgos ambientales asociados a la fracturación hidráulica

\begin{tabular}{ll}
\hline \multicolumn{1}{c}{ Riesgo ambiental } & \multicolumn{1}{c}{ Descripción } \\
\hline Uso intensivo de agua & Se emplean para un solo pozo cerca de 20 o00 m3, lo que afecta el consumo \\
& de agua en poblaciones vecinas al área de explotación, así como a la actividad \\
& agrícola. \\
& El agua se contamina en dos etapas: \\
& - Por explotación, debido a la mezcla con arena, sílice y otras sustancias químicas \\
Contaminación hídrica & durante la inyección a la roca. \\
& - Por falla en la integridad del pozo, lo que afecta los acuíferos. \\
Contaminación del aire y & El tratamiento de gas metano durante la técnica afecta la calidad del aire (efecto \\
contribución al cambio climático & invernadero). \\
Contaminación del suelo & Se contamina el suelo como consecuencia del agua de retorno, que contiene \\
& químicos y materiales radiactivos \\
& Se generan microsismos a causa de la presión ejercida para inyectar la solución \\
& acuosa. Como consecuencia, se materializa la comunicación entre un pozo y una \\
& zona no deseada.
\end{tabular}


Se observa en la tabla 1 que los impactos ambientales generados por la fracturación hidráulica se presentan desde la adecuación del terreno para la perforación del pozo, hasta la extracción del gas. Por esta razón se puede considerar que una práctica responsable de la alta dirección de una organización es analizar las problemáticas ambientales desde un sistema de gestión ambiental eficaz que las elimine o minimice con el fin de mejorar el desempeño.

Aquí se presenta la Norma Técnica Colombiana 14001: 2015, establecida por ICONTEC en 2015, como un estándar que reúne los requisitos mínimos para la conformación de un sistema de gestión ambiental. Para iniciar el proceso de implementación es importante llevar a cabo una primera revisión de estado (denominada revisión ambiental inicial), para determinar la situación actual de la organización y el desempeño ambiental en los últimos años. Debido a que la técnica de la fracturación hidráulica no convencional es nueva en Colombia, el diagnóstico ambiental inicial y el desempeño ambiental no tienen aplicación por falta de información respecto a los cumplimientos de los requisitos. Por ese motivo, la investigación tiene como propósito analizar los requisitos de la NTC ISO 14001:2015, para formular posibles soluciones a cada uno de estos requisitos y teniendo en cuenta las condiciones legales y técnicas de Colombia para las empresas de fracking hidráulico.

El documento se divide en tres secciones. La primera sección incluye la metodología para el desarrollo de la investigación. La segunda sección corresponde a los resultados; aquí se presenta cada requisito de la NTC ISO 14001:2015, su explicación y aplicación a las empresas de fracking. Por último, se abre la discusión sobre los hallazgos obtenidos.

\section{Método}

Esta investigación analiza los numerales que componen a la Norma Técnica Colombiana NTC ISO 14001:2015, con el fin de dar alternativas a la implementación de un sistema de gestión ambiental en empresas que practican fracking hidráulico de lutitas. La tabla 2 describe los requisitos solicitados por el estándar.

Tabla 2. Requisitos NTC ISO 14001: 2015

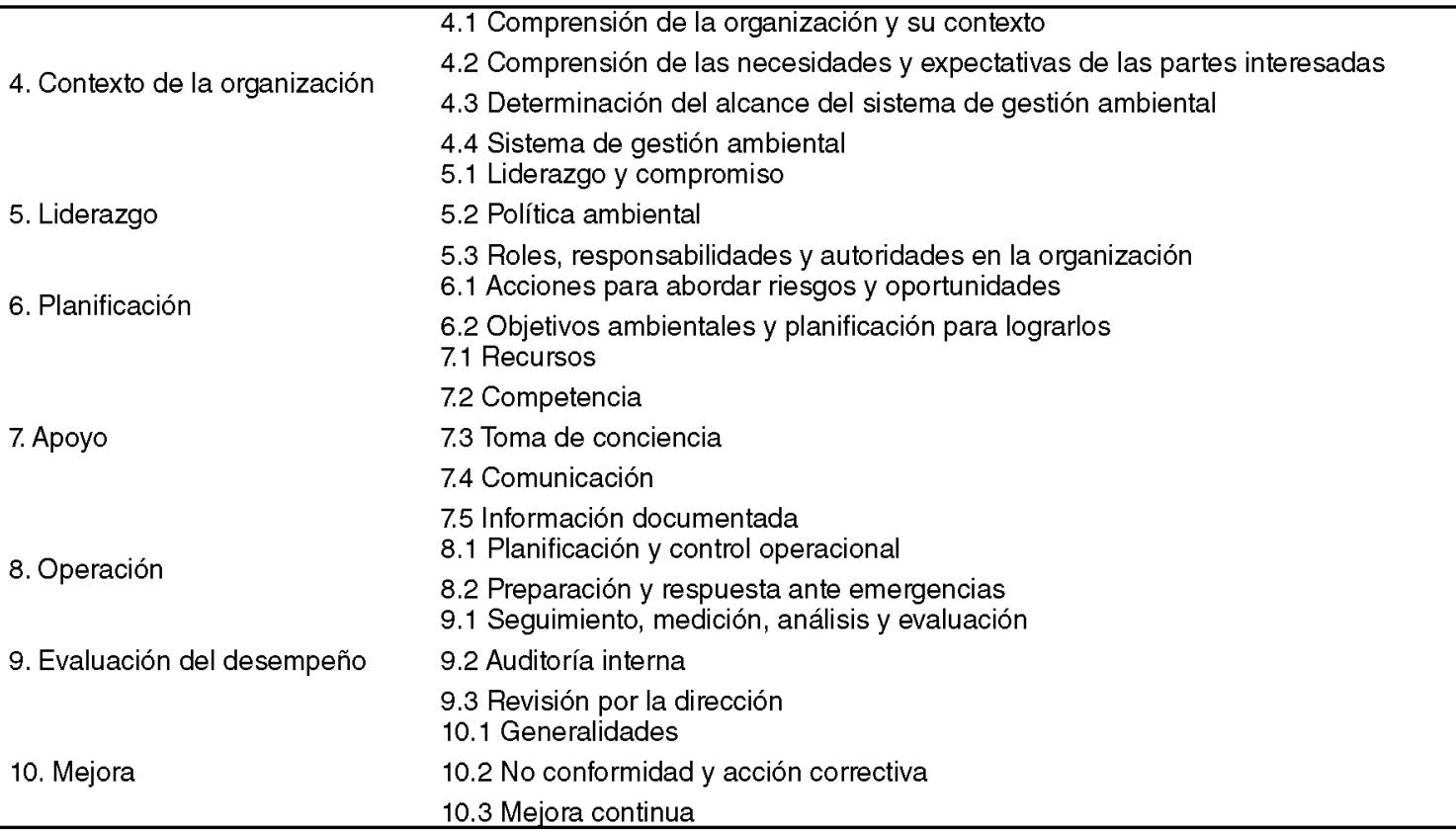

Fuente: ICONTEC (2015). 


\section{Resultados}

Se determinó para cada numeral de NTC ISO 14001:2015 las implicaciones que tendría la norma para el caso de empresas de fracturación hidráulica

\section{Capítulo 4. Contexto de la organización}

\section{Requisito 4.3 Determinación del alcance del sistema de gestión ambiental}

La organización es libre de definir el alcance del sistema de gestión ambiental (esto depende de los procesos o el tamaño de la empresa) (ICONTEC, 2015). Para una empresa en Colombia que realice fracturación hidráulica en yacimientos de gas shale el alcance debe ser para toda la organización.

\section{Requisito 4.4 Sistema de gestión ambiental}

Los requisitos generales que corresponden al numeral 4.4 se satisfacen al momento de implementar el sistema de gestión ambiental en la organización, aplicando el ciclo PHVA todo el tiempo para mejorar constantemente y definiendo y documentando el alcance del sistema con anterioridad.

\section{Capítulo 5. Liderazgo}

\section{Requisito 5.2 Política ambiental}

La política ambiental es la base de la gestión ambiental. De la política se derivan los objetivos y las metas ambientales que la organización se compromete a cumplir; por eso es fundamental que la alta dirección defina con base en su direccionamiento estratégico la política ambiental de forma completa y responsable y manteniendo el mismo camino de la política organizacional. Tal como lo afirma Clementes (1997): “La mejor manera de considerar una política medioambiental es pensar en términos de conservación de los recursos; en otras palabras, desperdiciar lo mínimo posible. E1 resultado es menor impacto medioambiental y mayor rentabilidad" (p. 74).

Con base en lo expuesto, la alta dirección de la organización puede establecer puntos claves ambientales a cumplir y puede identificar y plasmar el compromiso y la responsabilidad de la empresa con el medio ambiente. Igualmente, la política medioambiental debería responder los siguientes parámetros de la tabla 2 :

Tabla 2. Parámetros que la alta dirección debe responder al momento de redactar la política ambiental

\begin{tabular}{ll}
\hline & Compromiso de prevención \\
& Compromiso global de la organización respecto al medio ambiente \\
& Aplicación de la política y coordinación con el direccionamiento de la organización \\
Política medio ambiental & Compromiso de promover la gestión ambiental en todos los miembros de la organización \\
& Forma de cumplimiento a los requisitos ambientales y a las directrices de la política \\
Compromiso de atender situaciones de emergencia & Principios ambientales de la organización \\
\hline
\end{tabular}

La política ambiental de una empresa dedicada a la fracturación hidráulica en Colombia debe prevenir la contaminación e impulsar el uso eficiente de los recursos naturales en las localizaciones donde se lleve a cabo esta técnica, con el fin de mantener las condiciones ambientales constantes o, si es posible, mejorarlas (esto depende de las propiedades fisicoquímicas en las que se encuentre el medio ambiente). Un ítem fundamental para la política ambiental es el cumplimiento de los requisitos legales ambientales que se aplican en Colombia, así como de los requisitos que aporte la empresa operadora, la cual es responsable del campo en donde se va a llevar a cabo la fracturación. 


\section{Requisito 5.3. Roles, responsabilidades y autoridades en la organización}

La alta dirección se encarga de designar representantes responsables del sistema de gestión ambiental y de distribuir funciones específicas entre los puestos relevantes de la organización que participen en el desempeño ambiental de la empresa. De acuerdo con el Ministerio de Fomento (2005), algunas funciones ambientales a tener en cuenta en las empresas dedicadas al fracking son: - Coordinar el desarrollo y control de documentos del SGMA

- Mantener informada a la dirección sobre el funcionamiento del SGMA

- Garantizar la mejora continua del SGMA

- Dirigir el día a día de las cuestiones ambientales

- Realizar un seguimiento de la actuación medioambiental

- Asegurar el cumplimiento de la legislación

- Desarrollar la política medioambiental

- Fijar objetivos y metas

- Formación y sensibilización sobre medio ambiente

- Identificar expectativas ambientales de los clientes

- Determinar la actuación medioambiental de los proveedores

\section{Capítulo 6. Planificación}

De acuerdo con este capítulo, la organización tiene que establecer estrategias para identificar los aspectos ambientales, los requisitos legales y los otros requisitos. Además, se especifica que los objetivos, las metas y programas deben estar soportados en la política establecida por la alta dirección (ICONTEC, 2015).

\section{Requisito 6.1.2. Aspectos ambientales}

Para dar cumplimiento a este numeral, la organización puede establecer las actividades de cada fase de un proyecto de fracturación hidráulica. Las actividades generales en la operación se muestran en la tabla 3.

Tabla 3. Actividades para llevar a cabo una fracturación hidráulica para la extracción de gas shale

\begin{tabular}{|c|c|}
\hline Fase de construcción & Fase de explotación \\
\hline \multicolumn{2}{|l|}{ Ocupación de terreno } \\
\hline \multicolumn{2}{|l|}{ Despeje y desbroce } \\
\hline Movimeintos de tierra & Fractura hidráulica \\
\hline Uso de maquinaria pesada & Consumo de agua \\
\hline Nivelación de terreno & Torre de extracción \\
\hline Construcción torre de perforación y otra infraestructuras del & Uso de maquinaria pesada \\
\hline proyecto & Alamacenamiento de materiales y gestión de residuos \\
\hline Construcción de pozos (perforación horizontal y vertical) & Reducción de demanda energética \\
\hline \multicolumn{2}{|l|}{ Alamacenamiento de materiales y gestión de residuos } \\
\hline Demanda de mano de obra & \\
\hline
\end{tabular}

Fuente: Azuaga, González, Navas y Mirón (2012).

Una vez establecidas las actividades más específicas de la operación y definidas las actividades de otros procesos y condiciones normales, anormales y de emergencia de la organización, se procede a hallar los aspectos ambientales. En este momento la organización debe identificar en cada actividad los factores que pueden afectar negativa o positivamente al medio ambiente. Es posible 
que en una actividad se identifiquen los tres tipos de aspectos ambientales; esto depende de las prácticas preventivas ambientales que tenga la organización, así como del método para llevar a cabo las actividades. Los aspectos ambientales más significativos se muestran en la figura 2.

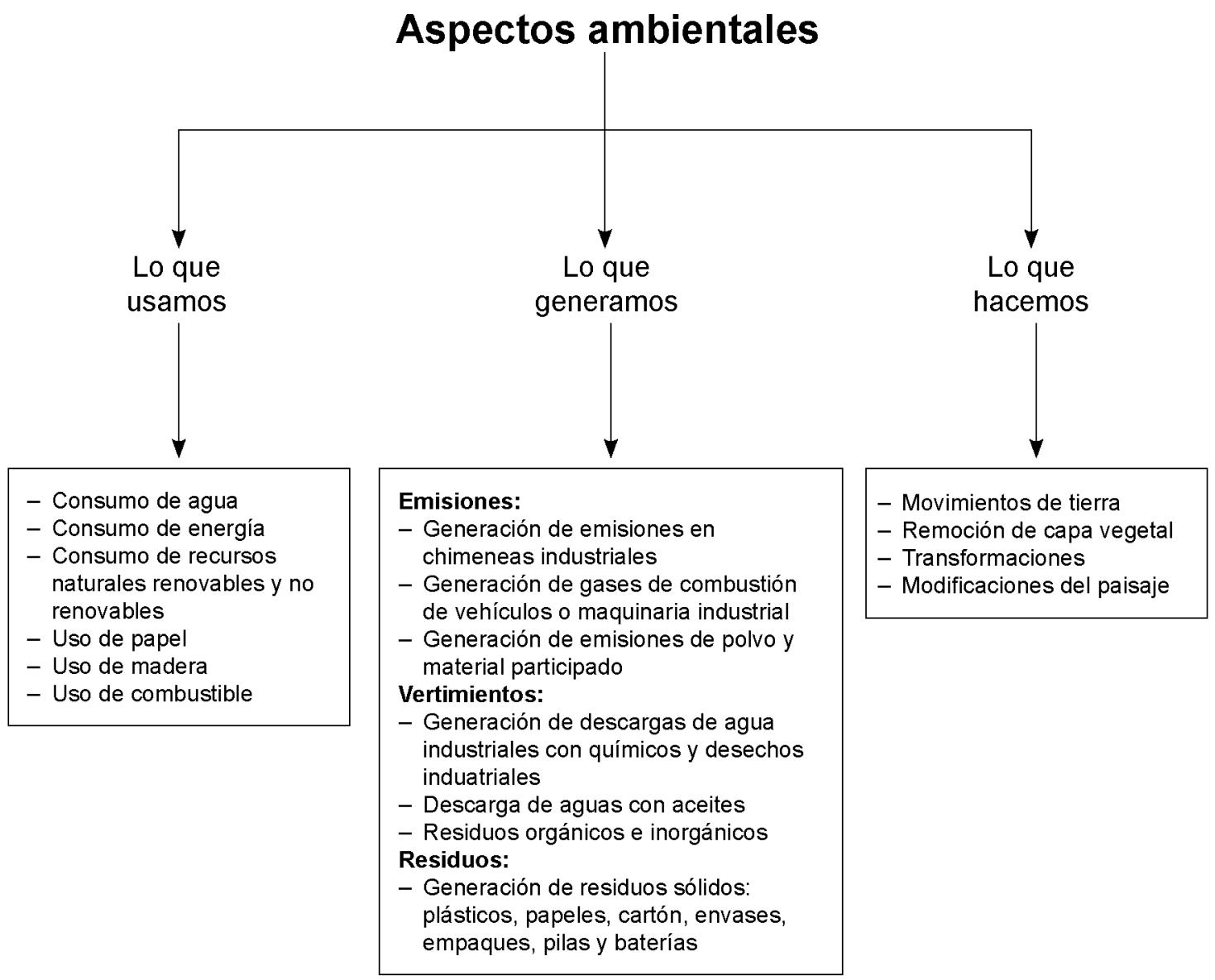

Figura 2. Aspectos ambientales aplicables a la fracturación hidráulica en lutitas.

Fuente: Pobeda (2005).

Después de identificar los aspectos ambientales de cada actividad de la organización que interactúa con el medio ambiente (ver figura 2), se procede a determinar el impacto positivo o negativo que cada actividad genera, con el fin de evaluar los aspectos ambientales significativos y desarrollar un rendimiento ambiental óptimo que no afecte las condiciones ambientales.

Un aspecto medioambiental significativo es aquel que tiene o puede tener un impacto medioambiental significativo. El proceso para la identificación de los aspectos medioambientales significativos asociados a las actividades de la organización trata de identificar aquellos que tienen mayor probabilidad de impacto significativo. (Ministerio de Fomento, 2005, p. 6).

En las tablas 3 y 4 se muestran algunos impactos ambientales para el análisis de aspectos ambientales significativos en las fases de construcción y explotación que están relacionadas al fracturamiento. 
Tabla 3. Impactos identificados en una construcción de shale gas

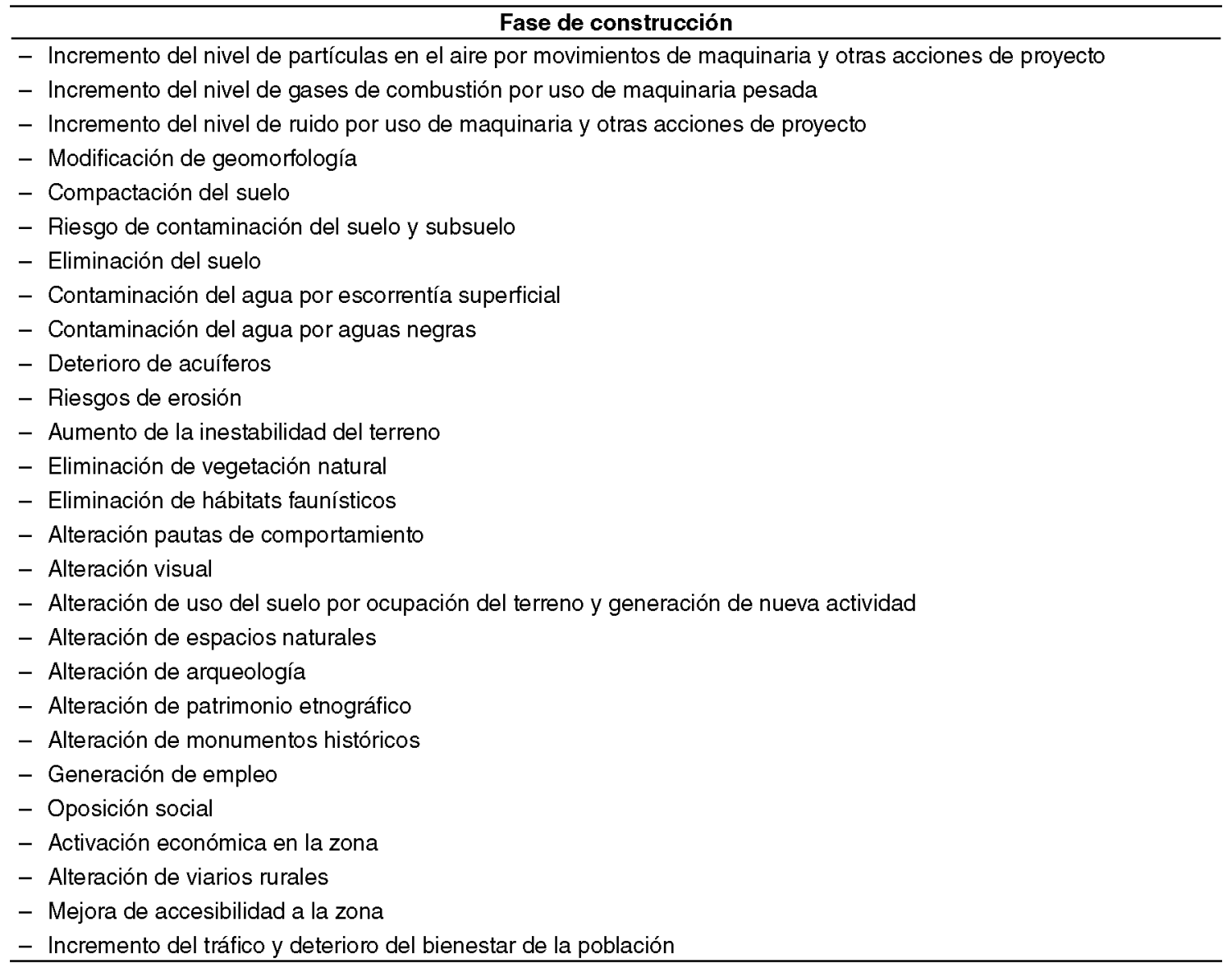

Fuente: Azuaga et ál. (2012)

Tabla 4. Impactos identificados en la explotación de shale gas

\begin{tabular}{l}
\hline \multicolumn{1}{c}{ Fase de explotación } \\
\hline Riesgo de contaminación de suelo y subsuelo por almacenamiento de productos químicos \\
Generación de aguas residuales y posible afección al entorno (aguas de fracking) \\
Consumo de agua \\
Contaminación de aguas subterráneas \\
Riesgo de producción de microsismos \\
Generación de empleos \\
Actividad económica en la zona \\
Disminución de dependencia energética externa \\
Riesgos para la salud de la población del entorno \\
Aumento del nivel del ruido por actividades de explotación
\end{tabular}

Fuente: Azuaga et ál. (2012).

\section{Requisito 6.1.3 Requisitos legales y otros}

Para que la organización implemente el compromiso de cumplimiento de los requisitos, es necesario conocer previamente todos los requisitos legales de carácter medioambiental que son aplicables, 
como también los requisitos que la empresa determinó como obligatorios (ICONTEC, 2015). Se puede revisar periódicamente estos requisitos, en especial los legales, para segura la sostenibilidad del cumplimiento. Los requisitos se dividen en tres categorías (ver figura 3):
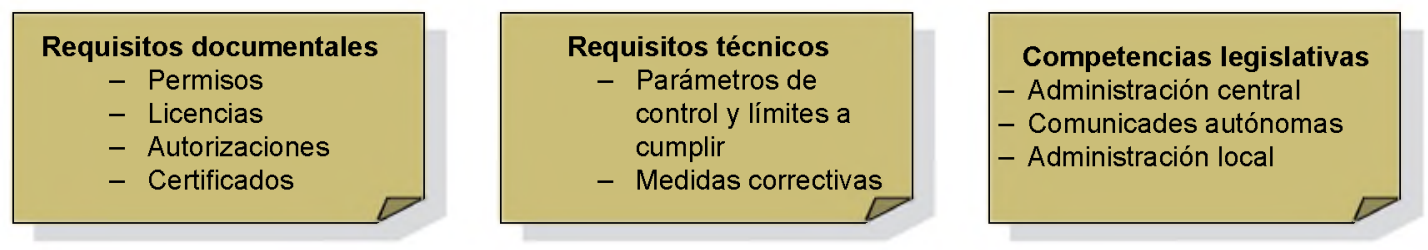

Figura 3. Requisitos que pueden afectar las actividades de una organización

Fuente: Ministerio de Fomento (2005).

Para el caso de una empresa que realice fracturación hidráulica en Colombia se pueden tener en cuenta los documentos legislativos que están explicados en la tabla 5.

Tabla 5. Requisitos legales aplicables al fracturamiento hidráulico

- Resolución 0909 de 2008, por la cual se establecen las normas y estándares de emisión admisibles de contaminantes a la atmósfera por fuentes fijas y se dictan otras disposiciones.

- Decreto 4741 de 2005, por el cual se reglamenta parcialmente la prevención y el manejo de los residuos o desechos peligrosos generados en el marco de la gestión integral.

- Decreto 3930 de 2010, por el cual se reglamenta en cuanto a usos del agua y residuos líquidos y se dictan otras disposiciones.

- Resolución 631 de 2015, por el cual se establecen los parámetros y los valores límites máximos permisibles en los vertimientos puntuales a cuerpos de agua superficiales y a los sistemas de alcantarillado público y se dictan otras disposiciones

- Decreto 2811 de 1974, por el cual se dicta el Código Nacional de Recursos Naturales Renovables y de Protección al Medio Ambiente.

- Ley 9 de 1979 por la cual se dictan medidas sanitarias.

- Decreto número 1076 de 2015, por medio del cual se expide el Decreto Único Reglamentario del Sector Ambiente y Desarrollo Sostenible.

- Ley 697 de 2001, mediante la cual se fomenta el uso racional y eficiente de la energía, se promueve la utilización de energías alternativas y se dictan otras disposiciones.

- Ley 373 de 1997, por la cual se establece el programa para el uso eficiente y ahorro del agua.

- Resolución 90341 de 2014, por la cual se establecen requerimientos técnicos y procedimientos para la exploración y explotación de hidrocarburos en yacimientos no convencionales.

- Resolución 0421 de 2014, por la cual se adoptan los términos de referencia para la elaboración del Estudio de Impacto Ambiental para los proyectos de perforación exploratoria de hidrocarburos y se toman otras determinaciones.

\section{Cumplimiento requisito 6.2 Objetivos ambientales y planificación para lograrlos}

Cuando la organización identifique los aspectos y los impactos ambientales de todas las actividades dentro del alcance del sistema de gestión ambiental y determine los requisitos legales y otros requisitos fundamentales para la práctica sostenible de la empresa desde una visión ambiental, se procede a formular objetivos y metas ambientales adecuados a la política ambiental de la organización y a su funcionamiento. Los programas ambientales permiten que la organización cumpla con los objetivos y las metas establecidas, destine los recursos necesarios y diseñe controles de acción eficaces (ICONTEC, 2015). 
Los objetivos ambientales permiten que la organización establezca un nivel de cumplimiento y de mejora en el sistema de gestión para un tiempo determinado. Cada objetivo mínimo puede incluir una meta ambiental, la cual es medible y cuantificable; por eso es importante que se implementen criterios de medición para determinar el cumplimiento de la organización (por ejemplo, indicadores de gestión).

Las organizaciones para establecer sus objetivos deberán tener en cuenta los siguientes puntos: - Los requisitos legales y otros requisitos aplicables

- Los aspectos/impactos ambientales significativos

- Las opciones tecnológicas

- Los condicionantes económicos, financieros, operacionales y comerciales

- La opinión de las partes interesadas

- Resultados de revisiones y auditorías anteriores. (Granero y Ferrando , 2007, p. 48).

Igualmente, la organización puede dar prioridad a los aspectos ambientales significativos al momento de establecer sus objetivos, para controlar, en primer lugar, las problemáticas directas de la organización con el medio ambiente y, en segundo lugar, destinar los recursos y las acciones necesarias para evitar impactos ambientales negativos que afecten el entorno. Las mediciones que se lleven a cabo en la organización deben representar el desempeño ambiental con base en los objetivos y en cumplimiento efectivo de las metas. La figura 4 ejemplifica un programa de gestión ambiental aplicado a un objetivo.

En la figura 4 se observa un ejemplo de programa de gestión ambiental para un aspecto ambiental específico, del cual se derivan tres objetivos ambientales y varias metas mediables. Estas organizaciones pueden utilizar una matriz para dar cumplimiento a todo el numeral de planificación, en la que se identifican los aspectos e impactos ambientales (se caracterizan con base en las partes interesadas), los requisitos legales y el nivel de impacto ambiental, con el fin de determinar los aspectos ambientales significativos y darles prioridad.

\section{Capítulo 7. Apoyo}

En este capítulo se determinan los recursos y las competencias del personal asociado al sistema de gestión ambiental, con el fin de poner en marcha el funcionamiento del sistema (ICONTEC, 2015).

\section{Requisito 7.1 Recursos}

Para dar cumplimiento a este ítem, la alta dirección de la organización puede desarrollar buenas prácticas con las que se asegure en todo momento la disponibilidad de recursos para el desarrollo del sistema de gestión ambiental. Los recursos ofrecidos pueden ser humanos, formativos, tecnológicos y financieros. También pueden presentarse otros recursos, como conocimiento especializado, contratación de externos, equipos de medida y controles, que pueden ser aplicables a la organización dedicada al fracturamiento hidráulico. 
Tabla 6. Ejemplo de programa de gestión ambiental para un objetivo concreto.

\begin{tabular}{|c|c|c|c|c|c|c|c|}
\hline Aspecto & Objetivos & Metas & Responsable & $\begin{array}{c}\text { Plazo } \\
\text { consecución }\end{array}$ & $\begin{array}{l}\text { Recursos/ } \\
\text { costes }\end{array}$ & $\begin{array}{c}\text { Estatus } \\
\text { actual }\end{array}$ & $\begin{array}{c}\text { Fecha de } \\
\text { consecución } \\
\text { objetivo }\end{array}$ \\
\hline \multirow{7}{*}{$\begin{array}{l}\text { Aguas } \\
\text { de } \\
\text { vertido }\end{array}$} & \multirow{3}{*}{$\begin{array}{c}\text { Asegurar el } \\
\text { vestido con pH } \\
\text { inferior a } 8,5\end{array}$} & $\begin{array}{l}\text { Seguimiento } \\
\text { diario de los } \\
\text { gráficos de } \mathrm{pH} \\
\text { y estudio de } \\
\text { causas de } \mathrm{pH} \\
\text { por encima de } \\
8.5\end{array}$ & $\begin{array}{l}\text { Energía y } \\
\text { fluidos }\end{array}$ & $\begin{array}{c}\text { Comienza julio } \\
05 \text { enero } 06\end{array}$ & Interno & Comenzado & Enero 06 \\
\hline & & $\begin{array}{l}\text { Contratación de } \\
\text { un organismo } \\
\text { de control para } \\
\text { realizar un } \\
\text { seguimiento } \\
\text { diario }\end{array}$ & Mantenimiento & $\begin{array}{c}\text { Comienza julio } \\
05 \text { enero } 06\end{array}$ & $54.000 €$ & Comenzado & \\
\hline & & $\begin{array}{l}\text { Mejorar y } \\
\text { aumentar los } \\
\text { controles de } \\
\text { fibra óptica para } \\
\text { tener un mayor } \\
\text { control sobre } \\
\text { emergencias }\end{array}$ & Mantenimiento & Agosto 05 & $1.500 €$ & Realizado & Agosto 05 \\
\hline & \multirow{3}{*}{$\begin{array}{c}\text { Disminuir los } \\
\text { parámetros de } \\
\text { SS1 y DQO2 }\end{array}$} & $\begin{array}{l}\text { Realizar un } \\
\text { seguimiento } \\
\text { de analíticas } \\
\text { externas } \\
\text { semanalmente } \\
\text { de vertido, } \\
\text { aceites y grasas }\end{array}$ & $\begin{array}{l}\text { Responsable } \\
\text { laboratorio }\end{array}$ & Noviembre 05 & $\begin{array}{l}\text { Incluido en } \\
\text { el contrato } \\
54.000 €\end{array}$ & En espera & $\begin{array}{l}\text { Noviembre } \\
05\end{array}$ \\
\hline & & $\begin{array}{l}\text { Estudiar la } \\
\text { entrada- } \\
\text { salida de } \\
\text { contaminación } \\
\text { y en función } \\
\text { de ello el mejor } \\
\text { método para } \\
\text { disminuir SS y } \\
\text { DQO }\end{array}$ & $\begin{array}{c}\text { Medio } \\
\text { ambiente }\end{array}$ & $\begin{array}{c}\text { Comienza julio } \\
05 \text { - septiembre } \\
05\end{array}$ & Interno & Comenzado & $\begin{array}{c}\text { Septiembre } \\
05\end{array}$ \\
\hline & & $\begin{array}{l}\text { Proceder a la } \\
\text { instalación del } \\
\text { proyecto elegido } \\
\text { si corresponde }\end{array}$ & & & & & \\
\hline & $\begin{array}{c}\text { Formación en } \\
\text { pretratamiento } \\
\text { de aguas de } \\
\text { vertido }\end{array}$ & $\begin{array}{l}\text { Realizar cursos } \\
\text { de formación } \\
\text { con empresas } \\
\text { externas en } \\
\text { mantenimiento } \\
\text { y operativa de } \\
\text { pretratamiento } \\
\text { de agua }\end{array}$ & RR. HH. & Enero 06 & $2.500 €$ & En espera & Enero 06 \\
\hline
\end{tabular}

Nota 1) Sólidos sedimentables; 2) demanda química de oxígeno

Fuente: Granero y Ferrando (2007). 


\section{Cumplimiento requisitos 7.2 y 7.3 Competencia y toma de conciencia}

La organización conoce las actividades para desarrollar un buen desempeño ambiental, así como las funciones y responsabilidades de los diferentes miembros de la empresa que participan en la gestión ambiental. En este punto lo que falta es determinar el nivel de competencia y formación para cada cargo. La toma de conciencia de todo el personal de la organización es fundamental para el éxito del sistema de gestión ambiental, porque de nada sirve llevar a cabo una planeación muy buena cuando el personal no sigue las directrices propuestas por la alta dirección, o no se evidencia un compromiso ambiental independientemente del puesto que ocupen en el organigrama de la empresa.

La toma de conciencia consiste en que cada persona comprenda las implicaciones ambientales que se derivan del rol que cumple en la organización y se responsabilice directamente con el entorno interno y externo que lo rodea.

Las organizaciones dedicadas a la fracturación hidráulica podrían desarrollar un plan completo de sensibilización, en el que el conocimiento de los aspectos ambientales significativos, las problemáticas que se generan por el incumplimiento de procedimientos y la responsabilidad de cada persona dentro del sistema de gestión ambiental sean puntos clave a tratar. Un ejemplo de plan de sensibilización aplicable para el caso de estudio se observa en la tabla 7.

Tabla 7. Ejemplo de un plan de sensibilización. Fuente: Ministerios de fomento (2005)

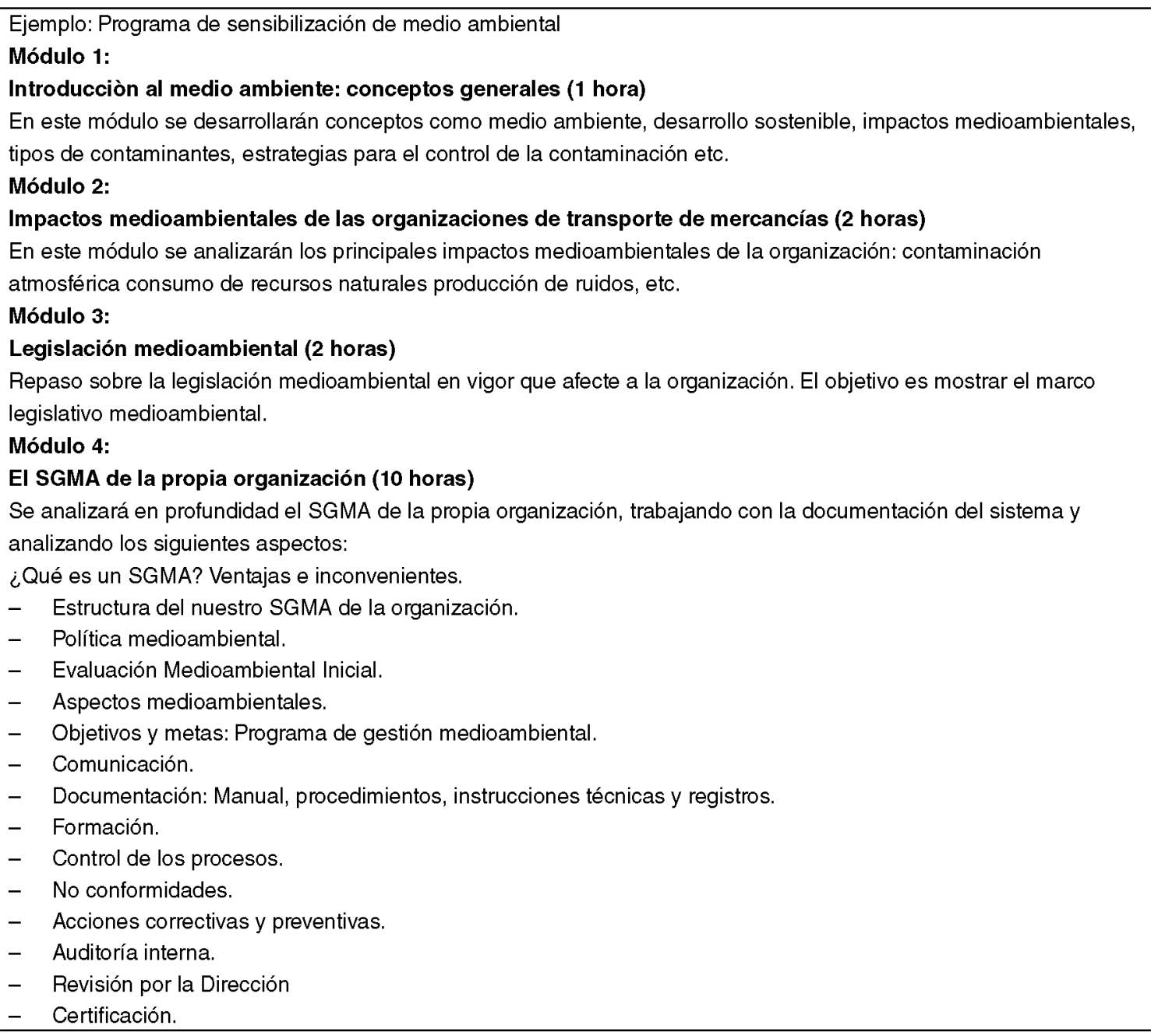


Todos los miembros de la organización requieren conocimientos generales sobre la gestión medioambiental dependiendo su rol en la empresa y la formación previa que tengan. Es en este caso en el que la organización puede diseñar un plan de formación de su personal desde una visión ambiental para asegurar la eficiencia del sistema de gestión ambiental desde el recurso humano.

En este ítem es importante especificar que:

No se trata únicamente de impartir contenidos formativos a través de la asistencia a cursos o jornadas, también es importante transmitir la importancia del cumplimiento de la política y los beneficios para el medio ambiente que un mejor comportamiento personal proporciona. (Zaro, 2002, p. 46)

\section{Requisito 7.4 Comunicación}

La organización puede establecer procedimientos para determinar las vías de comunicación de la información del sistema de gestión ambiental para todas las partes interesadas, como también puede diseñar registros para determinar la eficacia de los procedimientos (ICONTEC, 2015). En una organización se evidencia la comunicación interna y externa, en la que la finalidad es utilizar la información del sistema de gestión ambiental para tomar decisiones. Las vías de comunicación, tanto internas como externas, que pueden implementar las organizaciones dedicadas a la fracturación hidráulica se observan en la tabla 8.

Tabla 8. Vías de comunicación internas y externas de una organización.

\begin{tabular}{ll}
\hline & Empleados \\
& Boletines/revistas \\
& Tablero de anuncios \\
& Buzón de sugerencias \\
& Actividades extralaborales \\
& Reuniones con el comité/representantes \\
& Accionistas \\
Interna & Informes corporativos \\
& Informes de medio ambiente \\
& Contabilidad ambiental \\
& Proveedores \\
& Notificaciones \\
& Benchmarking \\
\hline & Administración \\
& Convenios, acuerdos y colaboraciones \\
& Grupos de trabajo y patrocinios \\
& Sociedad \\
& Diálogo activo con asociaciones \\
Informes públicos & Página web \\
Clientes \\
Respuesta a consultas \\
Estudios de comparación (competencia) \\
Folletos \\
Publicaciones sectoriales \\
\hline
\end{tabular}

Fuente: Granero y Ferrando (2007). 
La organización es libre de determinar las vías de comunicación pertinentes para el sistema de gestión ambiental. En el caso de las empresas dedicadas a la técnica de la fracturación hidráulica es fundamental una correcta comunicación con las partes interesadas externas, en especial con las comunidades aledañas a las localizaciones donde se desarrolla la operación para evitar inconvenientes o paros no programados debido a la inconformidad o al desconocimiento de las actividades que lleva a cabo la empresa para eliminar y minimizar los impactos ambientales negativos derivados de los aspectos significativos identificados.

\section{Requisito 7.5 Información documental}

\section{Requisito 7.5.2 Creación y actualización}

La organización puede crear y mantener al día toda la documentación necesaria para describir el sistema de gestión ambiental y describir qué hace para obtener un buen desempeño ambiental. La figura 4 ilustra los tipos de documentación.

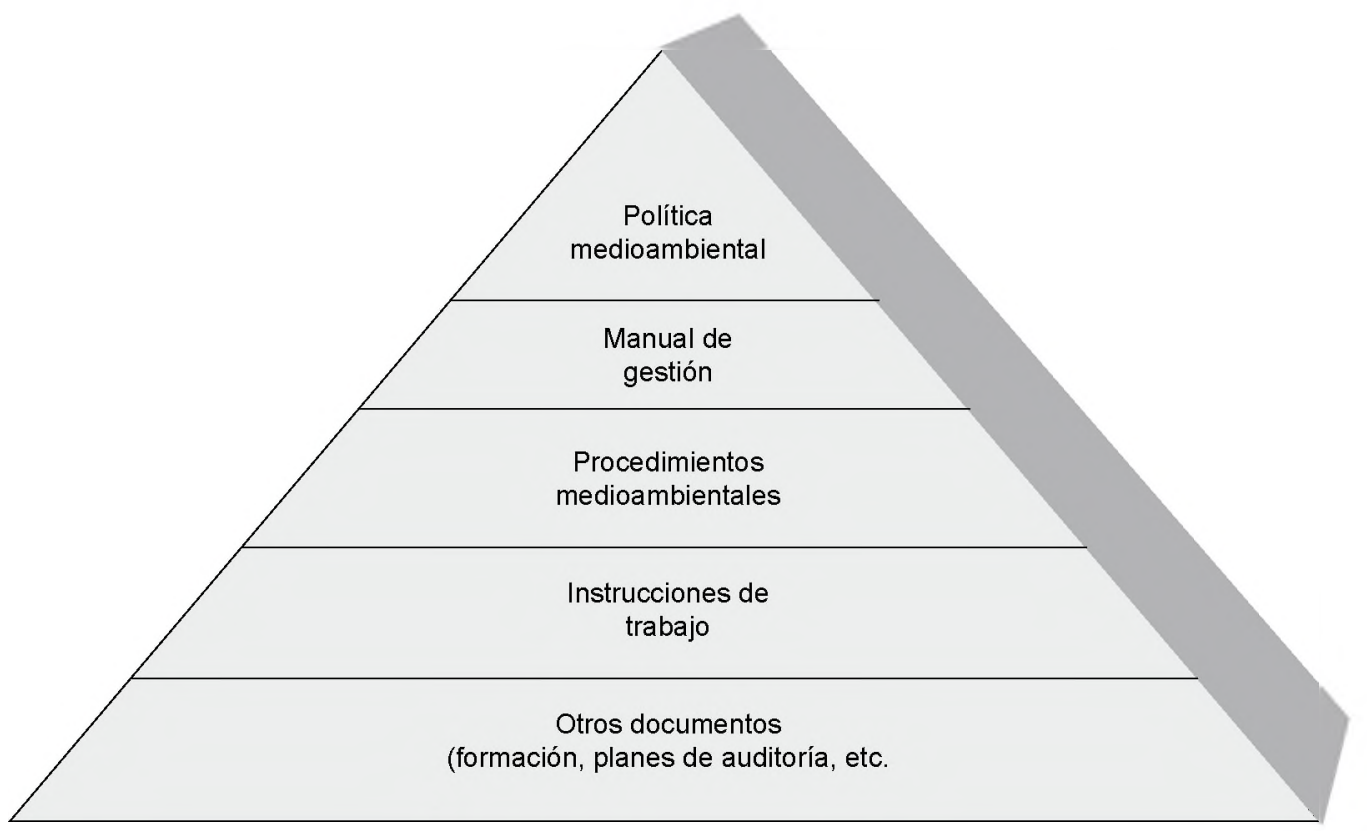

Figura 4. Estructura piramidal de organización de la documentación.

Fuente: Granero y Ferrando (2007).

Como se observa en la figura 4, la norma pide indirectamente un manual de gestión ambiental, el cual debe contener la debe contener: la política medioambiental liderada por la alta dirección, el alcance del sistema, la estructura organizacional de la empresa, las funciones y responsabilidades primordiales y sus interacciones.

En este ítem las organizaciones dedicadas a la fracturación hidráulica pueden diseñar diferentes documentos para la prevención de la contaminación y el uso eficiente de los recursos naturales.

En el último nivel de la documentación del sistema de gestión ambiental se encuentran los registros y todo archivo que no esté incluido en los anteriores niveles (como los planes de formación y sensibilización). 


\section{Requisito 7.5.3 Control de la información documentada}

Después de establecer la documentación para asegurar un comportamiento efectivo del sistema de gestión ambiental en la organización se procede a crear uno o varios procedimientos para controlarlos. Cuando las organizaciones dedicadas al fracturamiento implementen el sistema de gestión ambiental de forma correcta pueden dar evidencia de las conformidades con los requisitos de la NTC 14001:2004 por medio de los registros. Cabe resaltar que la organización puede tener evidencia del sistema de gestión ambiental por otro medio diferente a los registros.

Por lo tanto, cada organización "dispondrá de uno o varios procedimientos escritos donde describa cómo se da respuesta a la identificación, forma de conservación, protección, forma de recuperación, tiempo de archivo y la disposición de los registros del sistema de gestión ambiental" (Granero y Ferrando, 2007, p. 78).

Según Granero y Ferrando (2007, p. 79), los registros ambientales que genera la organización deben conservarse por un periodo de tiempo no inferior a los tres años (requisito de certificación), o por un periodo superior en el caso de que exista algún requisito legal aplicable a ese registro (por ejemplo, los documentos de control y seguimiento de residuos peligrosos deben archivarse durante cinco años).

\section{Capítulo 8. Operación}

En esta etapa la organización llevó a cabo la planificación del sistema de gestión ambiental con base en su política y a los aspectos ambientales para poder concluir con el programa de gestión ambiental (ICONTEC, 2015). En este momento la empresa debe realizar lo que planificó con anterioridad y partiendo de los requisitos de este numeral.

\section{Cumplimiento requisito 8.1 Control operacional}

En esta etapa la organización puede diseñar procedimientos o actividades con el fin de gestionar correctamente los aspectos ambientales y minimizar o eliminar los impactos negativos al medio ambiente. El control operacional debe tener en cuenta los siguientes ítems al momento de diseñar los procedimientos (ver figura 5).

Figura 5. Ítems claves para el control operacional.

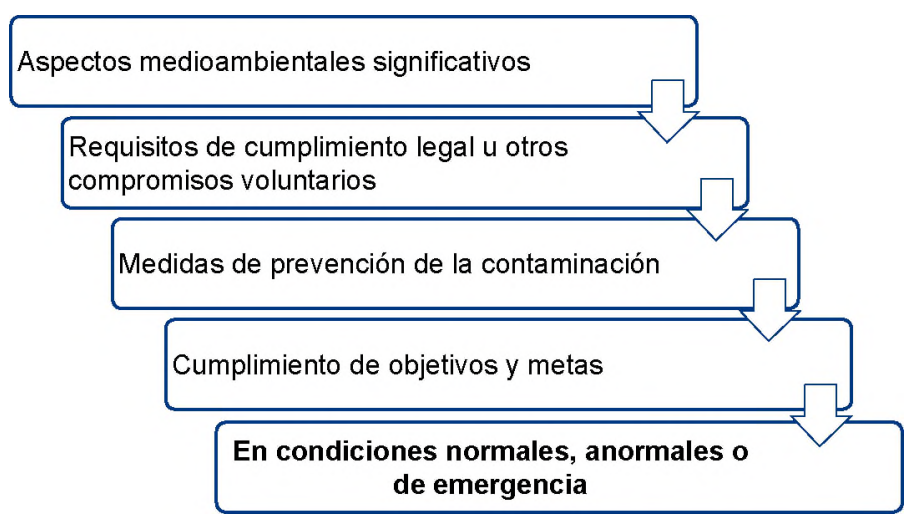

Fuente: Zaro (2002).

El control operacional que se dirija hacia un buen desempeño ambiental en la práctica del fracturamiento requiere que la organización asegure una minuciosa y completa planificación 
(numeral 6.1), porque esta es la base para diseñar los controles de las problemáticas ambientales. Algunos procedimientos aplicables a las empresas dedicadas a la fracturación hidráulica son: "Evaluación medioambiental de proveedores y subcontratistas, manipulación y almacenamiento de compuestos químicos tóxicos, gestión de residuos sólidos inertes, minimización de vertidos y emisiones, inicio y parada de actividades y cierre temporal de instalaciones" (Ministerio de Fomento, 2005, p. 24).

Debido a que en la operación se requiere de varios proveedores o subcontratistas, dependiendo de la localización y las condiciones físicas a las que se deba adaptar el equipo de trabajo, la organización puede incluirlos en su control operacional.

El control operacional debe ser extensivo a todas las operaciones, proveedores y subcontratistas en sus actividades relacionadas.

Es importante que los procedimientos de control operacional aplicables sean comunicados a proveedores y subcontratistas, así como mantener registros.

Además, si la empresa opta por incluir a los proveedores y subcontratistas medioambientales deberá establecer los criterios medioambientales exigibles y tener registros de la evaluación y de su mantenimiento (Ministerio de Fomento, 2005, p. 24).

\section{Requisito 8.2 Preparación y respuesta ante emergencias}

La organización es libre de implementar diferentes métodos para identificar situaciones de emergencia o accidentes que impliquen impactos ambientales negativos y poderlos controlar con el fin de reducirlos o eliminarlos en el momento que se presenten.

La organización deberá considerar, además: los métodos más apropiados para responder ante accidentes o situaciones de emergencia, como realizará la comunicación interna y externa, si la formación del personal es adecuada, las rutas de evacuación y puntos de encuentro, la posibilidad de situaciones de emergencia o accidente en instalaciones vecinas, etc. (Ministerio de Fomento, 2005, p. 69)

Algunos casos específicos que pueden generar accidentes o emergencia desde el punto de vista ambiental al momento de realizar la técnica son:

- Flujo de gas no deseado en superficie: puede variar las condiciones del aire en la localización y en las zonas aledañas.

- Pérdidas de circulación: pueden contaminar acuíferos someros y el suelo.

- Fallas en las válvulas de control del flujo de gas shale producido: emisiones no deseadas de gas mezclado con químicos del subsuelo y posibles incendios que afectan el aire.

\section{Cumplimiento requisito 9}

Evaluación del desempeño. Después de planear e implementar el sistema de gestión ambiental, la organización puede verificar el estado y el funcionamiento de las decisiones tomadas para determinar el desempeño ambiental.

\section{Requisito 9.1 Seguimiento, medición, análisis y evaluación}

La organización puede diseñar procedimientos para llevar a cabo el seguimiento y medición de los parámetros claves del sistema de gestión ambiental (ICONTEC, 2015). En este caso se podría implementar los indicadores de gestión en donde los documentos contienen la información sobre cómo tomar las medidas, establecer los parámetros específicos para la calibración y mantenimiento de equipos empleados en la medición y registrar la información pertinente a los resultados obtenidos. 
En este caso hay tres las líneas de acción:

- Seguimiento y medición de los parámetros clave del funcionamiento de las instalaciones y de las actividades relacionadas con aspectos significativos y el control operacional asociados a los mismos.

- Seguimiento y medición vinculados al control de la evolución de objetivos y de las actividades planificadas.

- Evaluación periódica del cumplimiento de la legislación y reglamentación aplicable (Zaro, 2002, p. 55).

Después de desarrollar los procedimientos documentados para la medición de parámetros, el mantenimiento y la calibración de equipos y establecer los registros de las mediciones, es importante determinar la periodicidad y los responsables del seguimiento del sistema de gestión ambiental. Al llevar a cabo el seguimiento y la medición establecidos se obtiene información real y confiable para determinar cuantitativamente el desempeño de la gestión de la organización.

\section{Requisito 9.1.2 Evaluación del cumplimiento}

La organización puede diseñar un procedimiento no necesariamente documentado para demostrar el cumplimiento de los requisitos legales y de los otros requisitos adoptados por la empresa. Cuando se lleven a cabo los procesos de seguimiento y medición relacionados con requisitos legales u otros requisitos, la organización podrá demostrar que efectivamente cumple con ellos. Para esto, puede editar un formato con la lista de requisitos legales y otros requisitos y completar uno a uno indicando cuándo, dónde y quién los ha verificado (Granero y Ferrando, 2007, p. 76). E1 objetivo del formato es asegurar que la organización tenga los registros pertinentes en los que se evidencie el cumplimiento de los requisitos.

\section{Requisito 9.2 Auditoria interna}

La organización puede elaborar uno o varios programas, los cuales tienen como fin establecer el o los objetivos de las auditorías, el alcance del programa (que en este caso es todo el sistema de gestión ambiental), los criterios, el tipo, la frecuencia y el número de auditorías a realizarse para obtener evidencia del cumplimiento de los requisitos de la NTC 14001:2015 y la eficacia de los procesos sistemáticamente.

Conociendo el número y la frecuencia de las auditorías internas aplicables para determinar el cumplimiento de los requisitos de la norma, la organización "elaborará uno o varios procedimientos de auditoria donde establezca claramente la cualificación requerida para los auditores, las responsabilidades, como planificar, preparar y realizar la auditoria y como mantener y comunicar los resultados obtenidos" (Granero y Ferrando, 2007, p. 79). Definidos los criterios de auditoria (que, en este caso, son el manual medioambiental y los procedimientos, los registros del sistema de gestión ambiental y la otra documentación) se realiza el plan de auditoría. Luego se designa el equipo auditor para que lleven a cabo una revisión inicial de la documentación proporcionada por la organización sobre su gestión ambiental y se lleva a cabo las actividades que componen la auditoria.

\section{Requisito 9.3 Revisión por la dirección}

La alta dirección de la organización puede realizar revisiones periódicas del sistema de gestión ambiental con el fin de establecer la eficacia de los procedimientos y las actividades implementadas, como también para direccionar al sistema hacia una mejora continua e identificar cambios o mejoras (ICONTEC, 2015). Con respecto a este numeral, Granero y Ferrado (2007) explican que: 
La alta dirección recopilará la información relevante desde el punto de vista del funcionamiento del sistema, para revisarlo a intervalos planificados y asegurarse de su correcta implementación. Esta información relevante puede estar formada por los resultados de auditorías, evaluaciones del cumplimiento legal y otros requisitos, las comunicaciones externas, el grado de cumplimiento de objetivos y metas, el estado de acciones correctivas y preventivas, etc. (p. 85)

La organización puede establecer un registro que evidencie el cumplimiento de este requisito como parte del control y para una posible certificación

\section{Capítulo 10. Mejora}

\section{Requisito 10.2 No conformidad, acción correctiva y acción preventiva}

La organización puede identificar fallas en el sistema de gestión ambiental, las cuales se originan por el incumplimiento de algún requisito de la NTC ISO 14001:2015. Se podría diseñar uno o varios procedimientos para detectar las no conformidades y determinar las causas potenciales, como también establecer informes de las no conformidades (especificando las acciones correctivas y preventivas aplicables).

\section{Discusión}

Se evidenció que la fracturación hidráulica para la explotación de hidrocarburos en yacimientos no convencionales, en este caso, gas contenido en roca shale, es una operación innovadora por las altas presiones que requiere, como también por el uso de recursos naturales, en especial agua. A esta técnica se le asocian varios impactos ambientales negativos, los cuales se pueden evitar o minimizar con un correcto desempeño ambiental basado en un sistema de gestión.

Sin embargo, se observa que la sola implementación de un sistema de gestión ambiental, como el caso de la NTC ISO 14001:2015, no es suficiente para que las empresas dedicadas a la fracturación hidráulicas manejen en su totalidad todas las posibles amenazas generadas por su actividad. Cabe resaltar que para lograr un desempeño ambiental óptimo se debe implementar varios controles de alta ingeniería, los cuales pueden tener un alto costo y afectar negativamente la rentabilidad de los proyectos.

Se demuestra así que la fracturación hidráulica en yacimientos no convencionales puede ser viable siempre y cuando se planifique la operación y se implementen los controles operacionales necesarios para eliminar o minimizar los impactos ambientales. Sin embargo, hay que aclarar que esta práctica se ve amenazada por situaciones externas referentes a la producción en países de Medio Oriente, que han desestabilizado los precios de petróleo, perjudicando la explotación no convencional, y que han afectado de manera indirecta la gestión de las empresas para mantener esta técnica de obtención de petróleo.

Es fundamental que las empresas operadoras y de servicios petroleros se direccionen hacia la sostenibilidad, para que sus actividades no solo se basen en el cumplimiento de requisitos, sino en el compromiso de cubrir sus obligaciones a nivel social, económico y ambiental en las localizaciones donde se trabaje, con tal de contribuir responsablemente con sus actividades al desarrollo de acciones futuras.

\section{RefEREncias}

Azuaga, M., González, A., Navas, L., y Mirón, A. (2012) Tecnologias y evaluación de impacto ambiental en la extracción de shale gas (tesis de maestría). Máster en Ingeniería y Gestión Medioambiental, Escuela de Organización Industrial, España. 
Bertinat, P., D’Elia, E., Ochandio, R., Svampa, M., Viale, E., y Observatorio Petrolero Sur. (2014). 20 Mitos y realidades del fracking ( $1^{\mathrm{a}} \mathrm{ed}$.). Buenos Aires: El colectivo.

Clementes, R. (1997). Guía completa de las normas ISO 14000. Barcelona: Gestión 2000.

Colombia Energía. (2012). Se extiende el boom de los no convencionales. Recuperado de http:// www.colombiaenergia.com/featured-article/se-extiende-el-boom-de-los-no-convencionales

Pobeda, P. (2005). Implementar un sistema de gestión ambiental según ISO 14001. Guía básica para las empresas comprometidas con el futuro. Bogotá: ICONTEC

Granero, J. y Ferrando, M. (2007) Cómo implantar un sistema de gestión ambiental según la norma ISO 14001: 2004. España: Confemetal.

ICONTEC. (2015). NTC ISO 14001:2015-Sistemas de gestión ambiental. Requisitos con orientación para su uso. Bogotá: Instituto Colombiano de Norma Técnicas y Certificación.

Ministerio de Fomento. (2005). Requisitos del SGMA según ISO 14001:2004. España: Ministerio de Fomento.

Robles, B. (2004). Impacto social y ambiental del fracking (1 $1^{a}$ ed.). México: Instituto Belisario Domínguez.

Unidad de Planeación Minero Energética. (2015). Evaluación de la contribución económica del sector de hidrocarburos colombiano frente a diversos escenarios de producción. Recuperado de: http://www1. upme.gov.co/sites/default/files/news/3828/files/resumen_fedesarrollo_mme_final.pdf

Zaro, L. (2002). Guía para la aplicación de la norma UNE-EN ISO 14001 en la PyME. España: CEPYME, Gobierno de Aragón y Asociación Española de Normalización y Certificación. 
